\title{
Les connaissances des enseignants universitaires algériens sur la notion de modèle scientifique
}

\section{The knowledge of Algerian university teachers on the notion of scientific model}

\author{
Djamel Houatis ${ }^{1 *}$ \\ ${ }^{1}$ Laboratoire de Physique des Techniques Expérimentales et ses Applications, Faculté des Sciences, \\ Université Yahia Fares de Médéa, Algérie.
}

\begin{abstract}
Résumé. Dans les activités du physicien, les ensembles indissociables théorie et modèle, expérimentation et modélisation sont omniprésents. La question de passage du concret à l'abstrait, de l'expérimentation à sa traduction formalisée passe obligatoirement par la prise en compte de "modèles". Cette composante pour un physicien est devenue très courante dans son discours scientifique, à tel point qu'il est devenu difficile à contourner. Dans cette étude, nous allons interroger un échantillon d'enseignants universitaires en exercice sur leurs conceptions et leurs utilisations des modèles. La grille d'analyse de nos résultats est basée sur des thèmes centraux préalablement sélectionnés. Les résultats de cette étude peuvent être pris en compte dans la conception des programmes afin de guider les enseignants à choisir une démarche appropriée d'enseignementapprentissage permettant de conduire leurs apprenants à la construction des modèles et à la pratique d'une activité de modélisation.
\end{abstract}

\begin{abstract}
In the physist's activities, the unseparated couples: theory/model and experimentation/modelling are omnipresent. The question of passing from the concrete to the abstract, from experimentation to its formalized translation; necessarily involves taking into account "models". In the educational context, this component is; therefore, unavoidable and indispensable in the process of acquiring and exposing knowledge. In this study, we will question a sample of university teachers about their conception and uses of models. The analysis grid for our results is based on previously selected central themes. The results of this study can be taken into consideration in the design of the programs in order to guide the teachers
\end{abstract}

* Corresponding author: houatis.djamel@univ-medea.dz 
to choose an appropriate teaching-learning approach leading their learners to the construction of the models and to the practice of a modelling activity.

\section{Introduction}

L'usage du vocable "modèle" est devenu, de nos jours, très fréquent dans le langage des sciences. "Nous ne raisonnons que sur des modèles" [1] affirmait déjà en son temps Paul Valery. Cette affirmation n'a jamais été aussi vraie qu'aujourd'hui. Ce terme est usité dans tous les champs disciplinaires. Observons qu'il n'a pas partout ni le même sens, ni encore moins le même statut.

La notion de modèle, dans les sciences modernes et contemporaines, a acquis une place prépondérante à tel point que l'on peut dire à la suite de Pascal Nouvel "Une étude qui ne peut déboucher sur un modèle est parfois regardée comme non scientifique, ou un problème qui ne se prête à aucune modélisation comme problème qui échappe à la science" [2]. Ou d'une façon encore plus radicale comme le pensait le mathématicien et philosophe Herbert Stachowiak qui affirmait en 1973 que "Par conséquent, toute la cognition est la cognition dans les modèles ou au moyen de modèles, et en général, toute rencontre humaine avec le monde a besoin d'un modèle comme médiateur" [3].

Christian Wissel fondateur de la modélisation écologique moderne en Allemagne exprime en 1989: "L'esprit humain est incapable de penser autrement qu'à travers les modèles" [4].

La notion de modèle bien que fort répandue dans tous les champs disciplinaires, possède en sciences physiques une spécificité toute particulière, et sa signification présente une résonnance et une portée propre à cette discipline. Nous nous intéresserons dans cette recherche plus particulièrement aux modèles en sciences physiques.

Dans le contexte scolaire, les modèles sont couramment utilisés dans les cours de physique pour aider à expliquer les concepts scientifiques; Ils ne sont pas nécessairement des objets d'enseignement mais sont souvent implicites dans les discours et les écrits en classe. L'importance que détiennent les modèles et les démarches de modélisation dans l'enseignement de la physique nous permet d'affirmer que la réussite d'un apprentissage est fonction de l'action efficace de l'enseignant et de sa méthode à diriger les activités des apprenants vers l'utilisation des modèles et de la modélisation en classe.

Des recherches antérieures sur l'appréciation des modèles par les enseignants ont montré qu'ils ont en général une compréhension épistémologique partielle de ces objets d'étude [511]. Cependant, pour comprendre pourquoi les modèles sont avantageux pour l'apprentissage des sciences, et quel rôle jouent-ils dans ce contexte? Il est nécessaire d'examiner comment les modèles sont utilisés et dans quel sens?

Nous nous proposerons donc dans cette étude d'examiner leurs usages par les enseignants universitaires algériens, c'est-à-dire quelles sont leurs manières de les introduire dans l'enseignement et de les mettre en œuvre et pour quels objectifs. Quelles fonctions des modèles mettent-ils en avant dans l'enseignement, quelles activités réalisent ils permettant de conduire les étudiants vers la nécessité de modèles (la modélisation)?

Il s'avère que de telles recherches sont encore rares pour l'enseignement supérieur. Les résultats de cette étude nous semble-t-il, être pris en compte dans la conception des programmes afin de guider les enseignants à choisir une démarche appropriée d'enseignement-apprentissage permettant de conduire leurs apprenants à la construction des modèles et à la pratique d'une activité de modélisation et d'acquérir différents modèles scientifiques. 


\section{Modèle et modélisation dans l'enseignement de la physique}

Le domaine des modèles et de la modélisation représente un vaste champ de recherche en épistémologie et en didactique des sciences, il a été exploré pendant des décennies et fait encore l'objet de recherches.

La physique a largement contribué à l'élaboration de la méthode scientifique basée entre autres sur l'expérimentation, le raisonnement et la formalisation. La tâche des physiciens a pour objectif de décrypter le comportement de la matière sous toutes ses formes. Les physiciens donc observent, mesurent, analysent, interprètent le comportement et les interactions de la matière et les rayonnements à travers l'espace et le temps. Ils utilisent des notions, s'aident de modèles, établissent des lois et des principes et développent des théories. En considérant les notions, les lois, les principes et les théories comme des modèles, les démarches suivies par les physiciens (comme tous les autres scientifiques) comprennent le plus souvent un travail de modélisation [12].

Dans l'activité de physicien, on distingue deux pôles : le champ empirique susceptible d'être étudié qui représente le monde des objets et des évènements... et le champ théorique susceptible d'être formalisé qui représente les lois et les théories (Fig. 1). Tout modèle peut être considéré comme un médiateur entre le champ théorique et le champ empirique [13-15]. On définit donc l'activité de modélisation comme étant la maitrise du problème de la mise en relation permanente entre données expérimentales et constructions théoriques [16].

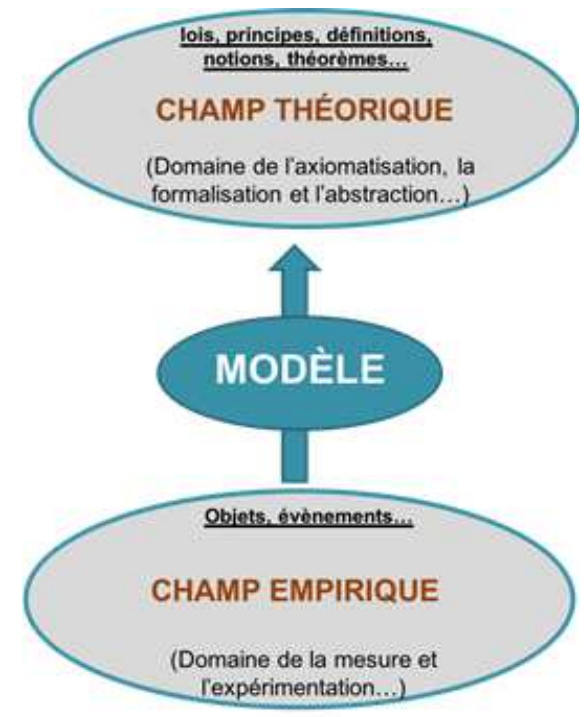

Fig. 1. Élaboration d'un modèle en physique.

Ainsi, le modèle n'est jamais un objet pris pour soi, il est toujours relationnel [17]. C'est un outil "pour penser" qui permet, d'un côté, d'extraire ou de sélectionner du champ empirique des objets comme : objets réduits, représentations imagées, textes, hypothèses, systèmes d'équations... qui substituent le réel complexe parfois inaccessible à l'expérimentation et, de l'autre côté, d'extraire du champ théorique des abstractions, des théorisations, des nouvelles représentations conceptuelles élaborées à partir des représentations préexistantes et des modèles simplifiées représentatifs du fonctionnement du réel. Selon S. Bachelard "le modèle renvoie à autre chose que lui-même et sa fonction est une fonction de délégation. Le modèle est un intermédiaire à qui nous déléguons la fonction de connaissance" [17]. Le scientifique observe le monde extérieur à travers des modèles qui lui permettent de l'appréhender et ce 
qui lui importe dans le modèle n'est pas son étude en soi mais plutôt son utilisation : comprendre, prévoir, calculer, rendre pensable ce qui est difficile à cerner...

En physique, certains concepts sont très complexes et difficiles à comprendre, compte tenu de l'absence d'une définition claire ou du degré élevé d'abstraction. La modélisation peut être un outil puissant pour interpréter ces types de concepts. Elle permet de ne pas rester silencieux devant ces phénomènes, ni de s'engager dans des représentations spontanées.

Dans la construction, l'adaptation et l'utilisation de modèles en classe, Martinand insiste à donner les trois caractéristiques essentielles aux modèles manipulés (et donc des processus de modélisation) [18] :

1. Ils sont et restent hypothétiques,

2. Ils sont modifiables pour s'adapter aux "besoins",

3. Ils sont pertinents pour certains problèmes dans certains contextes.

Selon l'usage de la modélisation en classe, on attribut généralement aux modèles quatre fonctions à mettre en avant:

1. Le modèle comme objet intermédiaire entre théorie et le phénomène,

2. Le modèle comme outil heuristique,

3. Le modèle comme outil de perception et de représentation,

4. Le modèle comme outil d'investigation scientifique et de mesure.

\section{Les conceptions de la notion de modèle physique par les enseignants à l'université.}

Dans ce qui suit, nous allons présenter les questions posées pour un échantillon d'enseignants universitaires en exercice sur leurs conceptions et leurs utilisations des modèles. S'appuyant sur une réflexion didactique mathématique, on peut soutenir que les conceptions des modèles par les enseignants jouent un rôle clé dans l'enseignement et l'apprentissage d'une discipline [19], notamment la physique.

Une attente raisonnable est que les modèles soient au centre de leur activité scientifique, les approches épistémologiques et didactiques de la notion de modèle soient acquises, et offrent des repères pour leur pratique scientifique et leur transposition didactique.

En dépit de son rôle central dans la conception et la conduite des situations d'enseignement, divers travaux de recherche indiquent que les enseignants ont souvent des difficultés dans la compréhension épistémologique de la notion de modèle [20-23].

\subsection{La construction du questionnaire}

Les données quantitatives ont été recueillies à l'aide d'un questionnaire (Annexe A) sur un échantillon d'enseignants en physique et en chimie de trois établissements universitaires algériens. L'Université Yahia Fares de Médéa (UYFM), l'Université Saâd Dahlab de Blida (USDB) et l'École Normale Supérieure de Kouba (ENSK).

La grille de questions utilisée a été construite en lien avec les quatre questions spécifiques de recherche suivantes:

1. Quelle place occupent les modèles scientifiques dans le discours et la pratique des enseignants?

2. Quelles définitions les enseignants attribuent-ils aux modèles?

3. Comment perçoivent-ils le rôle des modèles scientifiques?

4. Quelle stratégie adoptent-ils pour clarifier un modèle scientifique?

Le questionnaire comporte 10 items portant sur les axes suivants :

1. La nécessité du modèle.

2. La définition du modèle. 
3. Le rôle du modèle.

4. Les attentes des enseignants.

5. La pratique des enseignants pour l'enseignement du modèle.

Notre enquête sur terrain s'est déroulée, durant l'année universitaire 2017/2018. Après avoir été soumis à un groupe de 10 enseignants puis à un groupe de 20 enseignants parmi la population visée comme premier test permettant de voir la faisabilité de l'enquête, sa pertinence et son acceptabilité, le questionnaire a été soumis à un échantillon de 100 enseignants de physique et de chimie. Nous avons reçu en retour 66 questionnaires remplis, soit un taux de retour de $66 \%$, que nous pouvons considérer comme représentatif pour ce type d'enquête, répartis comme suit (Tableau 1.).

Table 1. Répartition des enseignants questionnés.

\begin{tabular}{|c|c|c|c|}
\cline { 2 - 4 } \multicolumn{1}{c|}{} & $\begin{array}{c}\text { Université } \\
\text { Yahia Fares } \\
\text { Médéa (UYFM) }\end{array}$ & $\begin{array}{c}\text { Université } \\
\text { Saad Dahlab } \\
\text { Blida(USDB) }\end{array}$ & $\begin{array}{c}\text { École Normale } \\
\text { Supérieure } \\
\text { Kouba(ENSK) }\end{array}$ \\
\hline $\begin{array}{c}\text { Nombre } \\
\text { d'enseignants }\end{array}$ & 35 & 09 & 22 \\
\hline Pourcentage & $53 \%$ & $14 \%$ & $33 \%$ \\
\hline
\end{tabular}

La description des principales caractéristiques de l'échantillon des enseignants universitaires est représentée par le tableau 2 suivant :

Table 2. Les caractéristiques de l'échantillon des enseignants.

\begin{tabular}{|c|l|c|c|}
\cline { 3 - 4 } \multicolumn{2}{|c|}{} & Fréquence & Pourcentage \\
\hline \multirow{2}{*}{ Sexe } & Féminin & 14 & $21 \%$ \\
\cline { 2 - 4 } & Masculin & 52 & $79 \%$ \\
\hline \multirow{2}{*}{ Discipline } & Physique & 42 & $64 \%$ \\
\cline { 2 - 4 } & Chimie & 24 & $36 \%$ \\
\hline \multirow{3}{*}{ Expérience en nombre d'années } & $\leq 4$ & 12 & $18 \%$ \\
\cline { 2 - 4 } & Entre 5 et 9 & 15 & $23 \%$ \\
\cline { 2 - 4 } & Entre 10 et 19 & 20 & $30 \%$ \\
\cline { 2 - 4 } & $\geq 20$ & 19 & $29 \%$ \\
\hline
\end{tabular}

\subsection{Résultats et discussion}

Afin d'analyser et d'interpréter les informations recueillies sur le terrain à travers le questionnaire, nous avons utilisés le logiciel SPSS. Ce dernier nous a permis d'appliquer les statistiques descriptives sur notre échantillon et de réaliser des tests statistiques indispensables à notre analyse.

Nous avons accordé une certaine importance aux résultats des réponses d'enseignants ayant une expérience professionnelle supérieure ou égale à 20 ans d'exercice, car ils disposent d'un capital scientifique et didactique plus important que d'autres, résultant au fil du temps de diverses activités interactives, stages, mobilisation des connaissances acquises et d'expérimentation pratique de l'enseignement ... À ce propos, nous émettrons donc l'hypothèse que les enseignants de cette catégorie ont d'abord des représentations idéales du métier et pour notre questionnaire également que les enseignants-débutants n'ont plus ou dans une moindre mesure. 


\subsubsection{L'axe relatif à la nécessité du modèle}

La notion de modèle et l'activité de modélisation sont constamment présentes dans l'activité des enseignants chercheurs des sciences empiriques notamment les sciences physiques et chimiques. Certains auteurs soutiennent que le raisonnement scientifique est dans une large mesure un raisonnement fondé sur des modèles [24].

L'objectif de ces items est de voir si les enseignants reconnaissent la nécessité des modèles dans leur enseignement, dans leur représentation des phénomènes étudiés et dans leur résolution des problématiques.

L'item 1.a nous informe si les enseignants utilisent systématiquement le terme modèle en classe. Les items 1.b et 1.c complètent l'item 1.a. Ils montrent le degré de corrélation des enseignants avec le modèle et de ses utilisations dans diverses situations. Les réponses à ces questions sont résumées dans le tableau 3 suivant :

Table 3. Résultats des items 1.a et 1.b et 1.c : La nécessité du modèle.

Item 1.a : Au cours de vos enseignements, utilisez-vous couramment le terme "modèle".

\begin{tabular}{|l|c|c|c|c|}
\hline & & Souvent & Rarement & Jamais \\
\hline UYF Médéa & $\mathrm{N}=35$ & $63 \%$ & $37 \%$ & $0 \%$ \\
\hline USD Blida & $\mathrm{N}=09$ & $67 \%$ & $33 \%$ & $0 \%$ \\
\hline ENS KOUBA & $\mathrm{N}=22$ & $64 \%$ & $27 \%$ & $9 \%$ \\
\hline Enseignants de physique & $\mathrm{N}=42$ & $67 \%$ & $33 \%$ & $0 \%$ \\
\hline Enseignants de Chimie & $\mathrm{N}=24$ & $59 \%$ & $33 \%$ & $8 \%$ \\
\hline Enseignants $\geq 20$ ans d'exercice & $\mathrm{N}=19$ & $84 \%$ & $16 \%$ & $0 \%$ \\
\hline Total & $\mathrm{N}=66$ & $64 \%$ & $33 \%$ & $3 \%$ \\
\hline
\end{tabular}

Item 1.b : Le lien entre modèle et phénomène susceptible d'être étudié.

\begin{tabular}{|l|c|c|c|c|}
\hline & & Réponse a & Réponse b & Réponse c \\
\hline UYF Médéa & $\mathrm{N}=35$ & $43 \%$ & $57 \%$ & $0 \%$ \\
\hline USD Blida & $\mathrm{N}=09$ & $44 \%$ & $56 \%$ & $0 \%$ \\
\hline ENS KOUBA & $\mathrm{N}=22$ & $55 \%$ & $45 \%$ & $0 \%$ \\
\hline Enseignants de physique & $\mathrm{N}=42$ & $50 \%$ & $50 \%$ & $0 \%$ \\
\hline Enseignants de Chimie & $\mathrm{N}=24$ & $42 \%$ & $58 \%$ & $0 \%$ \\
\hline Enseignants $\geq 20$ ans d'exercice & $\mathrm{N}=19$ & $68 \%$ & $32 \%$ & $0 \%$ \\
\hline Total & $\mathrm{N}=66$ & $47 \%$ & $53 \%$ & $0 \%$ \\
\hline
\end{tabular}

Item 1.c : Une problématique en physique trouve sa solution uniquement en la modélisant.

\begin{tabular}{|l|c|c|c|c|}
\hline & & Oui & Non & Réponse c \\
\hline UYF Médéa & $\mathrm{N}=35$ & $29 \%$ & $68 \%$ & $3 \%$ \\
\hline USD Blida & $\mathrm{N}=09$ & $56 \%$ & $44 \%$ & $0 \%$ \\
\hline ENS KOUBA & $\mathrm{N}=22$ & $50 \%$ & $45 \%$ & $5 \%$ \\
\hline Enseignants de physique & $\mathrm{N}=42$ & $45 \%$ & $53 \%$ & $2 \%$ \\
\hline Enseignants de Chimie & $\mathrm{N}=24$ & $29 \%$ & $67 \%$ & $4 \%$ \\
\hline Enseignants $\geq 20$ ans d'exercice & $\mathrm{N}=19$ & $53 \%$ & $47 \%$ & $0 \%$ \\
\hline Total & $\mathrm{N}=66$ & $39 \%$ & $58 \%$ & $3 \%$ \\
\hline
\end{tabular}

Les résultats montrent que la plupart des enseignants, de toutes les catégories, manifestent leur intérêt pour le modèle. Ils affirment avoir souvent utilisé ce terme dans leurs discours en classe. La moitié des enseignants de physique sont conscients de l'importance des modèles pour la représentation de tous les phénomènes susceptibles d'être étudié et de la résolution de n'importe quelle problématique en physique. Ainsi $84 \%$ des enseignants, ayant 20 ans et plus d'exercice, utilisent souvent le terme modèle dans leurs classes, $68 \%$ de cette catégorie d'enseignants estiment que tout phénomène physique peut être représenté par un modèle et 
$53 \%$ évoquent le modèle lorsqu'il s'agit de n'importe quelle problématique physique. Les enseignants de cette catégorie sont conscients -plus que d'autres- du rôle central des modèles pour la pratique scientifique. Ils reconnaissent la centralité des modèles dans la science à tous les niveaux, résultat conforme à ceux de Daniela $\mathrm{M}$. Bailer-Jones qui a interviewé des scientifiques britanniques au sujet de modèles scientifiques. Certains de ses scientifiques ont exprimé un parti pris excessif envers les modèles, ils prétendent qu'on ne peut pas échapper aux modèles et que toute la physique est construite essentiellement et constamment à travers des modèles [25]. On note que les étudiants ont affirmé, dans un questionnaire ${ }^{\dagger}$, avoir rarement entendu le terme modèle malgré son utilisation fréquente par leurs enseignants. Une telle situation didactique nous amène à nous interroger sur la manière dont les enseignants traitent les modèles en classe, et quel est le type de rapports spécifiques "contrat didactique" existant entre l'enseignant, l'apprenant et l'utilisation du concept de modèle.

\subsubsection{L'axe relatif à la définition du modèle}

Le concept de modèle est un concept polysémique [17], il peut avoir des significations très différentes, Il n'y a pas de consensus sur une définition unique de ce concept [26].

Notre principal intérêt dans ce thème est d'examiner comment les enseignants ont été eux même exposés à la notion de modèle. Quelles définitions attribuent-ils à cette notion ? Y at-il un écart entre leurs points de vue et ceux de leurs apprenants?

Nous avons présenté aux enseignants huit propositions qui pourraient être des définitions du concept de modèle, ensuite nous leur avons demandé de choisir quatre définitions qui correspondent le mieux à ce qu'ils entendent par modèle scientifique.

Les définitions sont classées en fonction du nombre de choix total donné par les enseignants. Nous avons constaté une certaine similarité dans les choix de toutes les catégories d'enseignants. Nous présentons ici les résultats des réponses de tous les enseignants (tableaux 4 et 5 ) et les résultats des réponses des enseignants ayant une période $\geq 20$ ans d'exercice (tableaux 6 et 7). Les résultats des réponses des autres catégories d'enseignants sont représentés dans l'annexe B.

Table 4. Résultats de l'item 2 : La définition du modèle pour tous les enseignants.

\begin{tabular}{|l|c|c|c|c|c|}
\hline \multicolumn{7}{|c|}{ Item 2 : Réponses de tous les enseignants } & $\mathrm{N}=66$. \\
\hline & Position 1 & Position 2 & Position 3 & Position 4 & Class. \\
\hline $\begin{array}{l}\text { Une représentation simplifiée de la } \\
\text { réalité d'un phénomène }\end{array}$ & $36 \%$ & $23 \%$ & $9 \%$ & $12 \%$ & 1 \\
\hline $\begin{array}{l}\text { Une représentation idéale de la } \\
\text { réalité d'un phénomène }\end{array}$ & $15 \%$ & $8 \%$ & $5 \%$ & $5 \%$ & \\
\hline Une formulation mathématique & $9 \%$ & $23 \%$ & $20 \%$ & $17 \%$ & 3 \\
\hline Un schéma simplificateur & $3 \%$ & $4 \%$ & $17 \%$ & $12 \%$ & 4 \\
\hline Une hypothèse. & $0 \%$ & $5 \%$ & $0 \%$ & $8 \%$ & \\
\hline $\begin{array}{l}\text { Un moyen de représentation utilisé } \\
\text { pour décrire et expliquer divers } \\
\text { phénomènes }\end{array}$ & $27 \%$ & $20 \%$ & $21 \%$ & $8 \%$ & 2 \\
\hline $\begin{array}{l}\text { Une matérialisation des énoncés de } \\
\text { la science dans un objet concret }\end{array}$ & $5 \%$ & $5 \%$ & $12 \%$ & $12 \%$ & \\
\hline
\end{tabular}

$\dagger$ D. Houatis, Le concept de modèle scientifique : vision des étudiants universitaires algériens, $3^{\text {ème }}$ édition du Colloque International sur la Formation et l'Enseignement des Mathématiques et des Sciences CIFEM'2020 26, 27 \& 28 Octobre 2020, CRMEF Casablanca-Settat, Section provinciale d'El Jadida, Maroc (2020) 
Un modèle est un système, conçu comme une représentation abstraite et utile d'un autre système.

\begin{tabular}{|l|l|l|l|l|}
\hline $3 \%$ & $3 \%$ & $11 \%$ & $14 \%$ & \\
\hline
\end{tabular}

Table 5. Principales définitions attribuées au modèle par tous les enseignants.

\begin{tabular}{|l|c|c|}
\hline $\begin{array}{c}\text { Principales définitions attribuées au modèle par tous les } \\
\text { enseignants questionnés } \mathbf{N = 6 6}\end{array}$ & Fréquence & Pourcentage \\
\hline Une représentation simplifiée de la réalité d'un phénomène & 53 & $80 \%$ \\
\hline $\begin{array}{l}\text { Un moyen de représentation utilisé pour décrire et expliquer } \\
\text { divers phénomènes }\end{array}$ & 50 & $76 \%$ \\
\hline Une formulation mathématique & 45 & $68 \%$ \\
\hline Un schéma simplificateur & 26 & $39 \%$ \\
\hline
\end{tabular}

Table 6. Résultats de l'item 2 : La définition du modèle pour les enseignants $\geq 20$ ans d'exercice.

\begin{tabular}{|l|c|c|c|c|c|}
\hline \multicolumn{7}{|c|}{ Item 2 : Réponses des enseignants $\geq 20$ ans d'exercice } & N=19. \\
\hline & Position 1 & Position 2 & Position 3 & Position 4 & Class. \\
\hline $\begin{array}{l}\text { Une représentation simplifiée de } \\
\text { la réalité d'un phénomène }\end{array}$ & $32 \%$ & $11 \%$ & $23 \%$ & $11 \%$ & 1 \\
\hline $\begin{array}{l}\text { Une représentation idéale de la } \\
\text { réalité d'un phénomène }\end{array}$ & $21 \%$ & $5 \%$ & $11 \%$ & $0 \%$ & \\
\hline Une formulation mathématique & $16 \%$ & $37 \%$ & $16 \%$ & $0 \%$ & 3 \\
\hline Un schéma simplificateur & $11 \%$ & $11 \%$ & $5 \%$ & $16 \%$ & 4 \\
\hline Une hypothèse. & $0 \%$ & $5 \%$ & $0 \%$ & $11 \%$ & \\
\hline $\begin{array}{l}\text { Un moyen de représentation } \\
\text { utilisé pour décrire et expliquer } \\
\text { divers phénomènes }\end{array}$ & $21 \%$ & $26 \%$ & $21 \%$ & $5 \%$ & 2 \\
\hline $\begin{array}{l}\text { Une matérialisation des énoncés } \\
\text { de la science dans un objet } \\
\text { concret. }\end{array}$ & $0 \%$ & $0 \%$ & $11 \%$ & $21 \%$ & \\
\hline $\begin{array}{l}\text { Un modèle est un système, conçu } \\
\text { comme une représentation } \\
\text { abstraite et utile d'un autre } \\
\text { système. }\end{array}$ & $0 \%$ & $0 \%$ & $11 \%$ & $0 \%$ & \\
\hline
\end{tabular}

Table 7. Principales définitions attribuées au modèle par les enseignants $\geq 20$ ans d'exercice.

\begin{tabular}{|l|c|c|}
\hline \multicolumn{1}{|c|}{$\begin{array}{c}\text { Principales définitions attribuées au modèle par les } \\
\text { enseignants } \geq \mathbf{2 0} \text { ans d'exercice N=19 }\end{array}$} & Fréquence & Pourcentage \\
\hline Une représentation simplifiée de la réalité d'un phénomène & 16 & $84 \%$ \\
\hline $\begin{array}{l}\text { Un moyen de représentation utilisé pour décrire et } \\
\text { expliquer divers phénomènes }\end{array}$ & 14 & $74 \%$ \\
\hline Une formulation mathématique & 13 & $69 \%$ \\
\hline Un schéma simplificateur & 9 & $47 \%$ \\
\hline
\end{tabular}

Les résultats montrent qu'une grande majorité des enseignants (plus de $75 \%$ ) conçoivent les modèles comme outil de représentation et de simplification. Les modèles mathématiques font partie des définitions les plus attribuées aux modèles par la plupart des enseignants (68\%).

Le modèle en tant qu'hypothèse ainsi que le caractère idéalisé et abstrait des modèles sont rejetés par un nombre plus important d'enseignants. Seuls 5 enseignants sur 35 de l'UYF Médéa et 1 seul enseignant sur 9 de l'USD Blida et 2 sur 22 de l'ENS Kouba ont évoqué le 
modèle comme hypothèse, de même, seuls 9 des 42 physiciens et 6 des 22 chimistes ont cité le modèle respectivement comme une abstraction et une idéalisation (Annexe B). Le tableau 8 donne les définitions du concept modèle les moins choisies par la totalité des enseignants et les enseignants ayant une période $\geq 20$ ans d'exercice.

Table 8. Définitions d'un modèle les moins choisis par les enseignants.

\begin{tabular}{|c|c|c|}
\hline $\begin{array}{c}\text { Définitions d'un modèle les moins choisies par les } \\
\text { enseignants }\end{array}$ & $\begin{array}{c}\text { Tous les } \\
\text { enseignants }\end{array}$ & $\begin{array}{l}\text { Enseignants } \geq 20 \\
\text { ans d'exercice }\end{array}$ \\
\hline Une représentation idéale de la réalité d'un phénomène & $32 \%$ & $37 \%$ \\
\hline Une hypothèse & $12 \%$ & $16 \%$ \\
\hline $\begin{array}{l}\text { Une matérialisation des énoncés de la science dans un } \\
\text { objet concret }\end{array}$ & $33 \%$ & $31 \%$ \\
\hline $\begin{array}{l}\text { Un modèle est un système, conçu comme une } \\
\text { représentation abstraite et utile d'un autre système }\end{array}$ & $30 \%$ & $11 \%$ \\
\hline
\end{tabular}

Les résultats indiquent que le potentiel de représentation, de schématisation, de simplification, de description, d'explication et de mathématisation apparaît comme un critère dominant pour qu'une proposition puisse être qualifiée comme une définition du modèle.

Les résultats indiquent également que les enseignants perçoivent que les définitions d'un modèle scientifique sont diverses. Ces résultats rejoignent en grande partie ceux rapportés dans des études antérieures notamment ceux obtenus par Van Driel et Verloop (1999) et de Bailer-Jones (2002) [6, 25].

\subsubsection{L'axe relatif aux rôles du modèle}

En tant que médiateur entre le champ théorique et le champ empirique, le modèle n'est jamais un objet pris pour soi, il est toujours relationnel. Cette caractéristique a permis d'affecter au modèle plusieurs fonctions.

L'objectif de cet item est de déterminer les principaux rôles que les enseignants accordent aux modèles scientifiques. Nous avons présenté huit propositions qui pourraient être des rôles du modèle, nous avons ensuite demandé aux enseignants de choisir quatre rôles qui correspondent le mieux à ceux avec lesquels ils se sentent le plus en accord.

Les rôles sont ensuite classés en fonction du nombre de choix total donné par les enseignants. Nous avons constaté une certaine similarité dans les choix de toutes les catégories d'enseignants. Les quatre propositions sélectionnées par toutes les catégories sont identiques, et ils ne varient que dans son ordre.

Nous présentons ici les résultats des réponses de tous les enseignants (tableaux 9 et 10) et les résultats des réponses des enseignants ayant une période $\geq 20$ ans d'exercice (tableaux 11 et 12). Les résultats des réponses des autres catégories d'enseignants sont représentés dans l'annexe C.

Table 9. Résultats de l'item 3 : Le rôle du modèle pour tous les enseignants.

\begin{tabular}{|l|c|c|c|c|c|}
\hline \multicolumn{5}{|c|}{ Item 3 : Réponses de tous les enseignants } & $\mathrm{N}=66$. \\
\hline & Position 1 & Position 2 & Position 3 & Position 4 & Class. \\
\hline $\begin{array}{l}\text { Prévoir avec une plus grande } \\
\text { probabilité et une meilleure } \\
\text { certitude, différents } \\
\text { comportements ou différentes } \\
\text { situations }\end{array}$ & $24 \%$ & $12 \%$ & $12 \%$ & $9 \%$ & 3 \\
\hline $\begin{array}{l}\text { Donner la description des } \\
\text { phénomènes }\end{array}$ & $30 \%$ & $18 \%$ & $14 \%$ & $6 \%$ & 2 \\
\hline
\end{tabular}




\begin{tabular}{|l|c|c|c|c|c|}
\cline { 2 - 5 } $\begin{array}{l}\text { Donner l'explication des } \\
\text { phénomènes }\end{array}$ & $20 \%$ & $14 \%$ & $8 \%$ & $12 \%$ & 4 \\
\hline $\begin{array}{l}\text { Avoir une fonction } \\
\text { d'investigation et de mesure }\end{array}$ & $2 \%$ & $3 \%$ & $14 \%$ & $9 \%$ & \\
\hline $\begin{array}{l}\text { Aider à la perception et à la } \\
\text { représentation des phénomènes. }\end{array}$ & $11 \%$ & $8 \%$ & $9 \%$ & $8 \%$ & \\
\hline $\begin{array}{l}\text { Mettre en relation des concepts } \\
\text { explicitement définis }\end{array}$ & $0 \%$ & $8 \%$ & $8 \%$ & $17 \%$ & \\
\hline $\begin{array}{l}\text { Aider à concevoir, à analyser ou } \\
\text { à imaginer des concepts. }\end{array}$ & $9 \%$ & $21 \%$ & $26 \%$ & $15 \%$ & \\
\hline $\begin{array}{l}\text { Aider à la conception } \\
\text { d'hypothèses et d'expériences. }\end{array}$ & $5 \%$ & $12 \%$ & $5 \%$ & $14 \%$ & \\
\hline
\end{tabular}

Table 10. Principaux rôles attribués au modèle par tous les enseignants.

\begin{tabular}{|l|c|c|}
\hline \multicolumn{1}{|c|}{$\begin{array}{c}\text { Principaux rôles attribués au modèle par tous les } \\
\text { enseignants questionnés } \mathbf{N = 6 6}\end{array}$} & Fréquence & Pourcentage \\
\hline Aider à concevoir, à analyser ou à imaginer des concepts & 47 & $71 \%$ \\
\hline Donner la description des phénomènes & 45 & $68 \%$ \\
\hline $\begin{array}{l}\text { Prévoir avec une plus grande probabilité et une meilleure } \\
\text { certitude, différents comportements ou différentes } \\
\text { situations }\end{array}$ & 38 & $58 \%$ \\
\hline Donner l'explication des phénomènes & 35 & $53 \%$ \\
\hline
\end{tabular}

Table 11. Résultats de l'item 3 : Le rôle du modèle, enseignants $\geq 20$ ans d'exercice.

\begin{tabular}{|l|c|c|c|c|c|}
\hline \multicolumn{5}{|c|}{ Item 3 : Réponses des enseignants $\geq 20$ ans d'exercice } & N=19. \\
\hline & Position 1 & Position 2 & Position 3 & Position 4 & Class. \\
\hline $\begin{array}{l}\text { Prévoir avec une plus grande } \\
\text { probabilité et une meilleure } \\
\text { certitude, différents } \\
\text { comportements ou différentes } \\
\text { situations }\end{array}$ & $16 \%$ & $5 \%$ & $5 \%$ & $16 \%$ & 3 \\
\hline $\begin{array}{l}\text { Donner la description des } \\
\text { phénomènes }\end{array}$ & $42 \%$ & $16 \%$ & $11 \%$ & $11 \%$ & 1 \\
\hline $\begin{array}{l}\text { Donner l'explication des } \\
\text { phénomènes }\end{array}$ & $21 \%$ & $26 \%$ & $16 \%$ & $5 \%$ & 2 \\
\hline $\begin{array}{l}\text { Avoir une fonction } \\
\text { d'investigation et de mesure }\end{array}$ & $0 \%$ & $11 \%$ & $21 \%$ & $5 \%$ & \\
\hline $\begin{array}{l}\text { Aider à la perception et à la } \\
\text { représentation des phénomènes. }\end{array}$ & $0 \%$ & $0 \%$ & $16 \%$ & $16 \%$ & \\
\hline $\begin{array}{l}\text { Mettre en relation des concepts } \\
\text { explicitement définis }\end{array}$ & $0 \%$ & $16 \%$ & $0 \%$ & $21 \%$ & \\
\hline $\begin{array}{l}\text { Aider à concevoir, à analyser ou à } \\
\text { imaginer des concepts. }\end{array}$ & $11 \%$ & $16 \%$ & $26 \%$ & $16 \%$ & 2 \\
\hline $\begin{array}{l}\text { Aider à la conception } \\
\text { d'hypothèses et d'expériences. }\end{array}$ & $21 \%$ & $11 \%$ & $5 \%$ & $0 \%$ & \\
\hline
\end{tabular}

Table 12. Principaux rôles attribués au modèle par les enseignants $\geq 20$ ans d'exercice.

\begin{tabular}{|l|c|c|}
\hline $\begin{array}{c}\text { Principaux rôles attribués au modèle par les enseignants } \\
\mathbf{2 0} \text { ans d'exercice N=19 }\end{array}$ & Fréquence & Pourcentage \\
\hline Donner la description des phénomènes & 15 & $79 \%$ \\
\hline
\end{tabular}




\begin{tabular}{|l|c|c|}
\hline Donner l'explication des phénomènes & 13 & $69 \%$ \\
\hline Aider à concevoir, à analyser ou à imaginer des concepts & 13 & $69 \%$ \\
\hline $\begin{array}{l}\text { Prévoir avec une plus grande probabilité et une meilleure } \\
\text { certitude, différents comportements ou différentes situations }\end{array}$ & 8 & $42 \%$ \\
\hline
\end{tabular}

Table 13. Rôles du modèle les moins choisis par les enseignants.

\begin{tabular}{|l|c|c|}
\hline \multicolumn{1}{|c|}{$\begin{array}{c}\text { Rôles du modèle les moins choisis par les } \\
\text { enseignants }\end{array}$} & $\begin{array}{c}\text { Tous les } \\
\text { enseignants }\end{array}$ & $\begin{array}{c}\text { Enseignants } \geq \mathbf{2 0} \\
\text { ans d'exercice }\end{array}$ \\
\hline $\begin{array}{l}\text { Aider à la perception et à la représentation des } \\
\text { phénomènes }\end{array}$ & $36 \%$ & $32 \%$ \\
\hline Aider à la conception d'hypothèses et d'expériences & $36 \%$ & $37 \%$ \\
\hline Mettre en relation des concepts explicitement définis & $33 \%$ & $37 \%$ \\
\hline Avoir une fonction d'investigation et de mesure & $28 \%$ & $37 \%$ \\
\hline
\end{tabular}

Les choix des enseignants montrent d'abord qu'ils sont conscients de la diversité des rôles de modèles scientifiques. En fait, la description, l'explication et la prévision sont des termes clés dans le choix de rôles des modèles. Ces résultats concordent largement avec ceux obtenus par Schwartz et Lederman (2005) dans leurs recherches associées aux points de vue de 24 scientifiques américains sur les modèles scientifiques [27]. Ces résultats montrent qu'une grande majorité des enseignants considère les modèles comme étant un outil intermédiaire entre « le monde des objets et des événements» et «le monde des théories ». Le choix de la proposition "Aider à concevoir, à analyser ou à imaginer des concepts" par $71 \%$ des enseignants renforce l'idée que, dans les allers-retours entre situations et modèles, l'enseignant reconnait le rôle fondamental du modèle qui reflète leur perception du fonctionnement de la physique. Ces résultats sont identiques à ceux d'une étude exploratoire menée par Patrick Roy et Abdelkrim Hasni (2014) auprès d'enseignants de sciences et technologies québécois du secondaire sur l'enseignement des modèles et de la modélisation [28]

Il convient de noter dans ce contexte que, parmi les apprenants, la distinction entre les champs empiriques des champs théoriques, et la prise en compte du modèle en tant qu'objet intermédiaire sont loin d'être acquises. Un travail de transposition didactique est donc nécessaire proposant à l'enseignant des outils pour qu'il puisse aider les apprenants à distinguer ces deux mondes. La recherche en didactique de la physique montre que ce sont justement ces allers-retours qui posent le plus de difficultés aux élèves et auxquels ils devront donc apporter une attention particulière [29].

La fonction de perception et de représentation est évoquée par un certain nombre d'enseignants (36\%) malgré son utilisation répandue en classe. Sans modèle, un phénomène peut passer complètement inaperçu. L'enseignant en classe, s'aide de vecteur ou de point matériel pour accéder aux phénomènes mécaniques et de rayons lumineux ou ondes pour accéder aux phénomènes optiques. Les modèles constituent donc des lentilles-conceptuelles [12].

La fonction heuristique du modèle est évoquée par les enseignants avec le même pourcentage ( $36 \%$ et $33 \%$ ). elle se manifeste dans la conception d'hypothèses et d'expériences et la mise en relation des concepts explicitement définis. L'enseignant ne peut pas explorer des phénomènes, concevoir d'hypothèses, faire abstraction, découvrir de nouveaux faits ou corréler des concepts en jeu s'il n'est pas guidé par un modèle.

Enfin, seuls $28 \%$ des enseignants attribuent au modèle la fonction d'investigation et de mesure (Tableau.13), elle est choisie par 4 enseignants sur 22 de l'ENS Kouba, 10 physiciens sur 42 et 8 chimistes sur 22 (Annexe C). Les enseignants des sciences expérimentales utilisent 
en permanence les mesures, qui sont présentes à tous les niveaux d'investigation. Elles sont nécessaires pour tester une hypothèse, quantifier les analyses, établir une loi, déterminer des paramètres et explorer le champ de validité d'un modèle. Dans une séance de travaux pratiques, les grandeurs et les mesures ont une place prépondérante, le tracé des courbes, l'interpolation des données et les tests statistiques sont inévitables, ce qui montre l'intérêt de cette fonction.

\subsubsection{L'axe relatif aux attentes des enseignants}

Selon Guy Brousseau, l'enseignant et l'apprenant sont liés par des phénomènes contractuels propres au fonctionnement de la relation didactique à propos d'objet d'enseignement/d'apprentissage [30]. L'un des problèmes majeurs de ce contrat est qu'il prive l'enseignant des conditions nécessaires à la compréhension et à l'apprentissage de la notion visée. L'objectif de ces items est d'étudier les attentes des enseignants à l'égard de leurs apprenants en ce qui concerne la conception et la construction de modèles scientifiques. Les réponses à ces questions sont résumées dans le tableau 14 suivant :

Table 14. Résultats des items 4.a et 4.b et 4.c : Les attentes des enseignants

\begin{tabular}{|c|c|c|c|c|}
\hline \multicolumn{5}{|c|}{ Item 4.a : Á votre avis, les étudiants sont-ils aptes à concevoir la notion de "modèle" ? } \\
\hline & & Oui & Non & Pas de réponse \\
\hline UYF Médéa & $\mathrm{N}=35$ & $69 \%$ & $31 \%$ & $0 \%$ \\
\hline USD Blida & $\mathrm{N}=09$ & $56 \%$ & $44 \%$ & $0 \%$ \\
\hline ENS KOUBA & $\mathrm{N}=22$ & $59 \%$ & $32 \%$ & $9 \%$ \\
\hline Enseignants de physique & $\mathrm{N}=42$ & $57 \%$ & $38 \%$ & $5 \%$ \\
\hline Enseignants de Chimie & $\mathrm{N}=24$ & $71 \%$ & $29 \%$ & $0 \%$ \\
\hline Enseignants $\geq 20$ ans d'exercice & $\mathrm{N}=19$ & $53 \%$ & $42 \%$ & $5 \%$ \\
\hline Total & $\mathrm{N}=66$ & $62 \%$ & $35 \%$ & $3 \%$ \\
\hline \multicolumn{5}{|c|}{$\begin{array}{c}\text { Item 4.b : Y'a-t-il un intérêt de définir ou de caractériser aux étudiants la notion de modèle et } \\
\text { modélisation? }\end{array}$} \\
\hline & & Oui & Non & Pas de réponse \\
\hline UYF Médéa & $\mathrm{N}=35$ & $94 \%$ & $0 \%$ & $6 \%$ \\
\hline USD Blida & $\mathrm{N}=09$ & $78 \%$ & $0 \%$ & $22 \%$ \\
\hline ENS KOUBA & $\mathrm{N}=22$ & $95 \%$ & $5 \%$ & $0 \%$ \\
\hline Enseignants de physique & $\mathrm{N}=42$ & $90 \%$ & $0 \%$ & $10 \%$ \\
\hline Enseignants de Chimie & $\mathrm{N}=24$ & $96 \%$ & $4 \%$ & $0 \%$ \\
\hline Enseignants $\geq 20$ ans d'exercice & $\mathrm{N}=19$ & $100 \%$ & $0 \%$ & $0 \%$ \\
\hline Total & $\mathrm{N}=66$ & $92 \%$ & $2 \%$ & $6 \%$ \\
\hline \multicolumn{5}{|c|}{$\begin{array}{c}\text { Item 4.c : Pensez-vous que les méthodes d'enseignement actuelles permettent à l'étudiant de se } \\
\text { construire la notion du modèle? }\end{array}$} \\
\hline & & Oui & Non & Pas de réponse \\
\hline UYF Médéa & $\mathrm{N}=35$ & $31 \%$ & $66 \%$ & $3 \%$ \\
\hline USD Blida & $\mathrm{N}=09$ & $33 \%$ & $67 \%$ & $0 \%$ \\
\hline ENS KOUBA & $\mathrm{N}=22$ & $23 \%$ & $77 \%$ & $0 \%$ \\
\hline Enseignants de physique & $\mathrm{N}=42$ & $26 \%$ & $72 \%$ & $2 \%$ \\
\hline Enseignants de Chimie & $\mathrm{N}=24$ & $33 \%$ & $67 \%$ & $0 \%$ \\
\hline Enseignants $\geq 20$ ans d'exercice & $\mathrm{N}=19$ & $11 \%$ & $89 \%$ & $0 \%$ \\
\hline Total & $\mathrm{N}=66$ & $29 \%$ & $69 \%$ & $2 \%$ \\
\hline
\end{tabular}

Dans le tableau ci-dessus, nous pouvons constater que les avis des enseignants de toutes les catégories sont convergents. Une grande majorité d'enseignant pense que les étudiants sont capables de concevoir la notion du "modèle", et qu'il est intéressant de définir ou de 
caractériser aux étudiants la notion du modèle et de la modélisation. Ainsi $100 \%$ des enseignants, ayant 20 ans et plus d'exercice, affirment que la perception du concept du modèle par les étudiants a des implications importantes sur les démarches d'enseignementapprentissage que les enseignants utilisent pour impliquer les élèves à s'approprier des modèles scientifiques. Malgré ces attentes, les résultats de notre recherche montrent que la majorité des enseignants de toutes les catégories (plus de 66\%), pensent que les "propositions didactiques" sur des contenus disciplinaire précis à enseigner n'est pas en faveur de l'appropriation de la notion de modèle, autrement dit, les apprenants ne sont pas placés dans les meilleures conditions pour construire la notion du modèles, comprendre leurs fonctions et leurs évolutions et d'identifier l'activité de la modélisation dans la démarche scientifique.

Les résultats montrent qu'il y a un écart entre les attentes des enseignants et ce qu'ils se manifestent en situation de classe, pour réduire cet écart, on suggère d'actualiser le processus de transposition en introduisant la notion du modèle dans les contenus scientifiques abordés.

Ces résultats confirment également la nécessité d'une formation, d'une part, des enseignants à l'enseignement de la notion du modèle, à sa réflexion épistémologique et, d'autre part, l'intégration des matières telles que l'histoire des sciences, l'histoire de la physique, l'épistémologie et la didactique dans les offres de formation licence et master et l'enseignement explicite de la notion du modèle et de la démarche de modélisation permettant ainsi de réduire considérablement cet écart.

\subsubsection{L'axe relatif à la pratique des enseignants pour l'enseignement d'un modèle scientifique}

L'élaboration d'un dispositif de formation nécessite l'analyse des pratiques des enseignants dans la classe [31]. Ces pratiques sont élaborées par l'enseignant lui-même à partir des difficultés qu'il rencontre et qui reflètent ses attentes. La notion du modèle possède une importance centrale dans la conception et la conduite des situations d'enseignement [32]. Comprendre les modèles scientifiques est une composante de la compréhension du fonctionnement réel de la science [27]. Or, de nombreux enseignants $(69 \%)$ indiquent en effet que les méthodes d'enseignement actuelles ne permettent pas à l'étudiant de se construire la notion du modèle (item 4.c). L'objectif de ces items est d'analyser quelques pratiques d'enseignants sur la mise en place de modèles dans la classe. Les résultats de l'item 5.a sont représentés dans le tableau 15.

Table 15. Résultats de l'item 5.a : Les modèles en tant que représentations multiples

\begin{tabular}{|l|c|c|c|c|}
\hline \multicolumn{5}{|c|}{ Item 5.a : Dans vos enseignements avez-vous utilisé des modèles différents ou contradictoires } \\
pour un même phénomène ?
\end{tabular}

Les résultats montrent que la plupart des enseignants (64\%) n'utilisent pas les modèles en tant que représentations multiples, ainsi $60 \%$ des physiciens et $71 \%$ des chimistes des trois 
universités déclarent éviter l'utilisation de nombreux modèles pour exprimer les caractéristiques d'un même phénomène. Cette pratique empêche l'apprenant de voir l'objet sous différents aspects ou formes, car de nombreux modèles représentent différentes vues, différentes versions du phénomène et illustrent également comment utiliser différentes informations.

Les modèles en tant que représentations multiples peuvent fournir une variété de perspectives et d'apparences et la plupart des apprenants les apprécient. De plus en reconnaissant la valeur de plusieurs modèles scientifiques pour un phénomène, on peut en déduire que les étudiants comprennent qu'un modèle n'est pas une réplique exacte et leur permet de faire la différence entre modèle et réalité. Cette activité donne également une bonne compréhension de la nature variable des modèles scientifiques qui était liée à la nature changeante des connaissances scientifiques.

Nous avons proposé aux enseignants six situations d'apprentissage, tout en demandant de choisir les situations adéquates pour clarifier un modèle scientifique. Les enseignants ont décrit l'implication des modèles dans leurs séquences d'enseignements de différentes manières (Tableau 16).

Table 16. Résultats de l'item 5.b : les stratégies adoptées par les enseignants

Item 5.b : Stratégies adoptées par les enseignants pour clarifier un modèle scientifique quelconque

\begin{tabular}{|l|c|c|c|c|c|c|c|}
\hline & $\begin{array}{c}\text { UYF } \\
\text { Médéa }\end{array}$ & $\begin{array}{c}\text { ENS } \\
\text { Kouba }\end{array}$ & $\begin{array}{c}\text { ENS } \\
\text { Kouba }\end{array}$ & $\begin{array}{c}\text { Enseign. } \\
\text { physique }\end{array}$ & $\begin{array}{c}\text { Enseign. } \\
\text { Chimie }\end{array}$ & $\begin{array}{c}\text { Enseign. } \\
\geq 20 \text { ans }\end{array}$ & Total \\
\hline $\begin{array}{l}\text { Exposer le modèle } \\
\text { en détail aux } \\
\text { étudiants qui } \\
\text { doivent se } \\
\text { l'approprier. }\end{array}$ & $40 \%$ & $89 \%$ & $55 \%$ & $50 \%$ & $54 \%$ & $53 \%$ & $52 \%$ \\
\hline $\begin{array}{l}\text { Procéder par } \\
\text { présentation } \\
\text { historique }\end{array}$ & $29 \%$ & $22 \%$ & $18 \%$ & $21 \%$ & $29 \%$ & $5 \%$ & $24 \%$ \\
\hline $\begin{array}{l}\text { Elaborer le } \\
\text { modèle à partir } \\
\text { des réponses des } \\
\text { apprenants avec } \\
\text { leurs mots et leurs } \\
\text { phrases. }\end{array}$ & $23 \%$ & $11 \%$ & $45 \%$ & $21 \%$ & $42 \%$ & $32 \%$ & $29 \%$ \\
\hline $\begin{array}{l}\text { Recourir à } \\
\text { l'épistémologie } \\
\text { du modèle. }\end{array}$ & $11 \%$ & $0 \%$ & $9 \%$ & $14 \%$ & $0 \%$ & $11 \%$ & $9 \%$ \\
\hline $\begin{array}{l}\text { Analyser et } \\
\text { interpréter le } \\
\text { modèle à partir } \\
\text { d'un problème } \\
\text { situé dans son } \\
\text { contexte. }\end{array}$ & $51 \%$ & $78 \%$ & $68 \%$ & $67 \%$ & $50 \%$ & $74 \%$ & $61 \%$ \\
\hline $\begin{array}{l}\text { Guider les } \\
\text { apprenants à } \\
\text { élaborer eux- } \\
\text { mêmes le modèle. }\end{array}$ & $60 \%$ & $11 \%$ & $9 \%$ & $40 \%$ & $29 \%$ & $37 \%$ & $36 \%$ \\
\hline
\end{tabular}

Les résultats montrent que les stratégies des enseignants pour clarifier un modèle scientifique se trouvent essentiellement centrées sur deux situations d'apprentissage : "Analyser et interpréter le modèle à partir d'un problème situé dans son contexte" $(61 \%)$ et "Exposer le modèle en détail aux étudiants qui doivent se l'approprier" (52\%). Dans les deux situations, 
les enseignants utilisent des modèles "constitués", ils n'évoquent pas explicitement la notion de modèle et ils ne réalisent aucune activité orientée vers la modélisation qui consiste à mettre en avant l'élaboration d'un modèle. Ce cadre didactique utilisé par les enseignants ne permet pas à l'étudiant d'être l'acteur de son apprentissage et ne contribuerait pas à donner du sens au modèle, les raisons et les conséquences du choix du modèle font défaut, et la réflexion sur les activités de modélisation est complètement absente. L'exposition, l'analyse et l'interprétation d'un modèle scientifique conçu ne résolvent pas le problème de confusion entre le monde des objets-événements et le monde des théories et modèle qui est essentiel pour l'apprentissage et la compréhension de cette notion.

Les situations d'apprentissage "Elaborer le modèle à partir des réponses des apprenants avec leurs mots et leurs phrases" et " Guider les apprenants à élaborer eux même le modèle" ont été sélectionnées par un nombre très limité d'enseignants $(29 \%$ et $36 \%)$. Ces deux situations permettent à l'étudiant de contribuer à la construction du modèle, lui permettant de comprendre le fonctionnement de la physique d'un point de vue de la modélisation. Elles ont tendance à expliquer, pour les apprenants, certains aspects de la notion de modèles scientifiques, ce qui leur permet d'apprendre comment les modèles jouent le rôle d'outils de la science, quelles sont "leurs conditions de validité, leurs utilisations possibles" et leur pouvoir heuristique. Ce choix réduit est peut-être dû aux difficultés rencontrées par les enseignants pour mettre en pratique ces stratégies.

L'apprentissage épistémologique et historique du concept de modèle contribue de manière significative à la clarification de cette notion. Certains chercheurs en didactique des sciences [33-37] se sont concentrés sur l'introduction de l'épistémologie et de l'histoire des sciences dans l'apprentissage en tant qu'approche pédagogique innovante ayant un impact positif sur l'acquisition des concepts scientifiques. En effet les pratiques des enseignants pour l'enseignement d'un modèle scientifique ne prennent que très peu en charge ces deux dimensions (épistémologique $9 \%$, historique $24 \%$ ) présentant souvent un savoir construit et abouti, de façon anhistorique et dépourvu de toute réflexion épistémologique. Les résultats montrent qu'aucun des enseignant de l'USD Blida et aucun des chimistes des trois universités n'adopte la situation d'apprentissage "Recourir à l'épistémologie du modèle". Seulement 5\% d'enseignants ayant une expérience $\geq 20$ ans d'exercice choisissent la situation "Procéder par présentation historique". L'intégration des dimensions épistémologiques et historiques dans la préparation de leurs enseignements est pratiquement absente.

Une solide connaissance de l'épistémologie et de l'histoire de la discipline s'impose à l'enseignant qui aborde les rapports entre modèles, théories, champs empiriques et champs théoriques, les questions de domaines de validité et les fonctions des modèles. De même le recours à ces disciplines joue un rôle motivant pour les apprenants et facilite réellement les apprentissages scientifiques notamment la notion de modèle.

Ces résultats montrent également que les pensées et les conceptions épistémologiques des enseignants sur les modèles sont très limitées et que leurs connaissances historiques sont partielles. Les résultats des études antérieures confirment cette déficience dans une large mesure [5-11]. Afin de donner aux enseignants des clarifications sur les fondements épistémologiques et historiques liés aux modèles et la modélisation, la conception et la mise en place des dispositifs de formation est une nécessité $[10,38,39]$. Ce programme de formation vise à développer des compétences professionnelles des enseignants pour l'enseignement de ces objets d'étude.

Divers chercheurs soulignent l'importance d'une culture épistémologique et historique pour les enseignants de sciences pour mener à bien des actions didactiques efficaces et pour l'acquisition de nouvelles postures professionnelles [40-43]. 


\section{Conclusion et perspectives}

Le concept de modèle est constamment présent dans la pratique des enseignants, il ne constitue pas un objet d'enseignement à part entière, mais il est souvent implicite dans les discours et les écrits en classe.

La perception des modèles par les enseignants reflète une compréhension simple et indistincte de modèles moins élaborés que les conceptions scientifiques, leurs connaissances en matière de modèles et de la modélisation se sont révélées variées et limitées. Cependant, la plupart des enseignants physiciens et chimistes des trois universités algériennes reconnaissent l'importance de modèle en tant qu'outil majeur dans le processus scientifique et didactique.

Les enseignants sont également conscients de la polysémie du terme modèle et de la diversité de ses fonctions. Ils le perçoivent principalement comme outil de schématisation, de simplification, de description, d'explication, de prédiction et de mathématisation. Les modèles sont peu conceptualisés comme des hypothèses ou des représentations idéales ou abstraites. La fonction heuristique du modèle et la fonction d'investigation et de mesure sont rarement reconnus par les enseignants.

Les résultats montrent également qu'il y a un écart entre les attentes des enseignants et ce qu'ils se manifestent en situation de classe, cet écart peut être réduit en actualisant le processus de transposition par l'implication explicite de la notion du modèle et de l'activité de modélisation dans les contenus scientifiques enseignés.

Les modèles sont perçus par les enseignants comme essentiels pour enseigner et pour faire de la science. Cela peut être utilisé comme une incitation à utiliser la démarche de modélisation en classe, et signifie également que les épistémologues et les didacticiens des sciences doivent prendre au sérieux les modèles et en faire un thème central de l'enseignement de la physique et de la chimie et améliorer les connaissances de la modélisation

Les choix des enseignants concernant leurs pratiques montrent que les contenus des enseignements des sciences physiques et chimiques à l'université n'incitent pas à l'utilisation de la modélisation.

Il ressort de l'étude des pratiques des enseignants que les matières scientifiques telles que la physique et la chimie sont traditionnellement introduite en classe, l'accent y est mis sur les méthodes d'organisation, de présentation, d'analyse et d'interprétation des phénomènes et moins sur les théories/modèles.

Les enseignants ne se placent pas généralement dans les meilleures conditions pour clarifier la notion du modèle :

1. Ils n'utilisent pas les modèles en tant que représentations multiples.

2. Ils n'analysent et n'interprètent et n'explorent uniquement des modèles déjà construit et abouti.

3. Ils ne réalisent aucune activité orientée vers la modélisation qui consiste à mettre l'étudiant comme acteur de la construction de son modèle.

4. Ils n'ont pas recours à la dimension épistémologique et historique dans la préparation de leurs enseignements.

Nous soutenons la nécessité, d'une part, d'une transposition de savoir permettant d'articuler un cadre didactique et un cadre épistémologique pour construire des faits didactiques liés à l'enseignement du concept du modèle et, d'autre part, d'une formation des enseignants en épistémologie et histoire des sciences. Cette formation permet à l'enseignant de développer ses compétences professionnelles, voire même d'acquérir de nouvelles compétences, de renouveler ses pratiques pédagogiques et de développer son aptitude à des réflexions historico-épistémologiques et à contribuer de façon active à l'évolution des connaissances relatives aux savoirs pour enseigner. Elle donne aussi la possibilité aux enseignants de se mettre dans des conditions favorables à mener les apprenants à comprendre 
les activités des chercheurs et des praticiens des sciences, la manière dont ceux-ci construisent et utilisent leurs modèles, le statut de ces modèles, leurs sens, leurs fonctions, leurs limites et leurs enjeux épistémologiques.

Je remercie vivement tous les membres du comité scientifique et d'organisation de la $3^{\text {ème }}$ édition du colloque international CIFEM'2020, je tiens à remercie particulièrement les évaluateurs de la première et de la deuxième phase pour leurs lectures et corrections et leurs précieux commentaires. J'adresse également un merci spécial à mon directeur de laboratoire (LPTEA) le Pr Zitouni Hannachi pour son soutien et ses recommandations judicieuses.

We thank the General Directorate of Scientific Research and Technological Development (DGRSDT), Algiers, Algeria for the financial support.

\section{Références}

1. P. Valéry, Cahiers (1894-1945). p.835 (Encyclopédie de la Pléiade, Gallimard, Paris, 1975).

2. P. Nouvel, Enquête sur le concept de modèle. (PUF, Paris, 2002)

3. H. Stachowiak, Allgemeine Modelltheorie. p.56 (Springer, Wien, 1973)

4. R. Müller, The concept of model: Definition and types (2006). Available at: http://www.muellerscience.com/ENGLISH/ Theconceptofmodel.definitions.htm>.

5. J. Smit, M. Finegold, Models in physics: Perceptions held by final-year prospective physical science teachers studying at South African universities. International Journal of Science Education, 5, 17, 621-634 (1995)

6. J. H. Van Driel, N. Verloop, Teachers' knowledge of models and modelling in science. International Journal of Science Education,11, 21, 1141-1153 (1999)

7. J. H. Van Driel, N. Verloop, Experienced teachers' knowledge of teaching and learning of models and modelling in science education. International Journal of Science Education, 12, 24, 1255-1272 (2002)

8. R. S. Justi, J. K. Gilbert, Science teachers' knowledge about and attitudes towards the use of models and modelling in learning science. International Journal of Science Education, 12, 24, 1273-1292 (2002)

9. R. S. Justi, J. K. Gilbert, Teachers' views on the nature of models. International Journal of Science Education, 11, 25, 1369-1386 (2003)

10. B. A. Crawford, M. J. Cullin, Supporting prospective teachers' conceptions of modeling in science. International Journal of Science Education, 11, 26, 1379-1401 (2004)

11. I. Henze, J. H. Van driel \& N. Verloop, Science teachers' knowledge about teaching models and modelling in the context of a new syllabus on public understanding of science. Research in Science Education, 37, 2, 99-122 (2007)

12. I. A. Halloun, Modeling theory in science education (Kluwer Academic Publishers, Dordrecht, Holland, 2004)

13. I.T. Koponen, Models and Modelling in Physics Education: A Critical Re-analysis of Philosophical Underpinnings and Suggestions for Revisions. Science \& Education, 78, 16, 751-773 (2007)

14. A. Tiberghien, Modeling as a basis for analyzing teaching-learning situations. Learning and instructions, 4, 71-87 (1994)

15. G. Sensevy, A. Tiberghien, J. Santini, S. Laubé \& P. Griggs, An epistemological approach to modelling: case studies and implication for science teaching. Science Education, 92, 424-446 (2008)

16. M. Bunge, Philosophie de la physique. (Seuil, Paris,1975)

17. S. Bachelard, Quelques aspects historiques des notions de modèle et de justification des modèles. In P. Delattre \& M. Thellier (Eds.), Elaboration et justification des modèles. 09-20 (Maloine, Paris, 1979) 
18. J.-L. Martinand, Schémas didactiques pour la modélisation en sciences et technologies. Spectre, 1, 40, 20-24 (2010)

19. R.P. Philipp, Mathematics Teachers' Beliefs and Affect. In F. K. Lester (Ed.), Second Handbook of Research on Mathematics Teaching and Learning: A Project of the National Council of Teachers of Mathematics, 1, 257-317 (National Council of Teachers U.S.A, 2007)

20. J. Désautels, M. Larochelle, B. Gagne \& F. Ruel, La formation à l'enseignement des sciences : le virage épistémologique. Didaskalia, 1, 49-67 (1993)

21. G. Robardet, La formation des enseignants de sciences physiques et le mythe naturaliste. In M. Caillot (dir.) Actes du quatrième séminaire de recherche en didactique des sciences physiques. 4-22 (Université de Picardie Jules Vernes (CURSEP) et IUFM de Picardie, 1994)

22. R. Porlàn Ariza, E. Garcia Garcia, A. Rivero Garcia \& R. Martin Del Pozo, Les obstacles à la formation professionnelle des professeurs en rapport avec leurs idées sur la science, l'enseignement et l'apprentissage. Aster, 26, 207-235 (1998)

23. K. J. Gilbert, Models and modelling: routes to more authentic science education. International Journal of Science and Mathematics Education, 2, 115-130 (2004)

24. N. R. Giere, Using Models to Represent Reality. Proceedings of the International Conference on Model-Based Reasoning in Scientific Discovery, held December 1998, 17-19, (Springer Science, Business Media, LLC, Pavia, ltaly 1998)

25. D. M. Bailer-Jones, Scientists' thoughts on scientific models. Perspectives on Science, 3, 10, 275-301 (2002)

26. A.M. Drouin, Les modèles en questions, Aster, 7, Modèles et modélisation, (INRP, Paris 1998)

27. R.S. Schwartz, N.G. Lederman, What scientists say: Scientists' views of models? Paper presented at the annual Meeting of the American Educational Research Association (AERA, Montreal, Canada, 2005)

28. P. Roy, A. Hasni, Les modèles et la modélisation vus par des enseignants de sciences et technologies du secondaire au Québec. McGill Journal of Education, 492, 349-371 (2014)

29. P. Gaidioz, J. A. Vince \& A. Tiberghien, Privilégier la modélisation: des outils d'enseignement pour aider l'élève. Bulletin de l'Union des Physiciens, (2004)

30. G. Brousseau, Le contrat didactique : le milieu. Recherche en didactique des mathématiques, 9,3, 309-336 (1990)

31. E. Sanchez, M. Prieur \& D. Devallois, Fonder la formation sur l'analyse des pratiques, besoins exprimés versus besoins réel : le cas de l'enseignement des sciences de la Terre au lycée. Colloque AIFRISS, (Bordeaux, 2004)

32. R. G. Justi, J. Van Driel, The development of science teachers' knowledge on models and modelling: promoting, characterizing, and understanding the process. International Journal of Science Education, 5,27, 549-573 (2005)

33. F. Abd-el-khalick, N. Lederman, The influence of the history of science courses on students'views of the nature of science. Journal of Research in Science Teaching, 10, 37, 1057-1095 (2000)

34. M. Guedj, Utiliser les textes historiques dans l'enseignement des sciences physiques. Didaskalia 26, 75-95 (2005)

35. H. Gispert, N. Hulin, M.C., Robic, Science et enseignement. L'exemple de la grande réforme des programmes des lycées au début du XXème siècle. Préface de Pierre Caspard, 360 p. (Vuibert, INRP, Lyon, Paris, 2007)

36. C. De Hosson, L'histoire des sciences : un laboratoire pour la recherche en didactique et l'enseignement de la physique. Enseignement de la physique [physics.ed-ph]. (Université Paris-Diderot - Paris VII, 2011) 
37. L. Maurines, L'histoire des sciences comme outil de recherche et d'enseignement : exemples des couleurs obtenues par passage de la lumière à travers un prisme. Actes des quatrièmes journées de l'ARDIST, 133-239 (ARDIST, Lyon, 2005)

38. L. Danusso, I. Testa \& M. Vicentini, Improving prospective teachers' knowledge about scientific models and modelling: Design and evaluation of a teacher education intervention. International Journal of Science Education, 7,32, 871-905 (2010)

39. C.V. Schwarz, Y.N. Gwekwerere, Using a guided inquiry and modeling instructional framework (EIMA) to support preservice K-8 science teaching. Science Education, 1, 91, 158-186 (2007)

40. G. Lacombe, Pour l'introduction de l'histoire des sciences dans l'enseignement du second cycle. Aster, 5, 87-116 (1987)

41. L. Guilbert, D. Méloche, L'idée de science chez les enseignants en formation : un lien entre l'histoire des sciences et l'hétérogénéité des visions? Didaskalia, 2, 7-30 (1993)

42. G. Fourez, V. Englebert-Lecomte \& P. Mathy, Nos savoirs sur nos savoirs. Un lexique d'épistémologie pour l'enseignement (De Boeck, Bruxelles, 1997)

43. M. Madrane, M. Khaldi \& M. Talbi, Exploitation didactique de l'histoire des sciences dans une perspective de formation à l'enseignement des sciences. Didaskalia, 31, 167189 (2007). 


\section{ANNEXE A}

\section{QUESTIONNAIRE}

Ce questionnaire s'adresse aux enseignants de physique et de chimie à l'université, il a pour objectif d'étudier comment les enseignants perçoivent la notion de modèle, leur position et la façon d'adopter et d'utiliser ce concept. Nous vous prions de bien vouloir remplir ce questionnaire, en choisissant la bonne réponse parmi plusieurs propositions.

Nombre d'années d'enseignement:

Lieu :

1- Au cours de vos enseignements, utilisez-vous couramment le terme "modèle" ?

$\begin{array}{ll}\text { a- } & \text { Souvent } \\ \text { b- } & \text { Rarement } \\ \text { c- } & \text { Jamais }\end{array}$

2- Le lien entre modèle et phénomène susceptible d'être étudié.

a- $\quad$ Tous ces phénomènes peuvent être représentés par un modèle.

b- Uniquement quelques phénomènes

c- Autres réponses ou justifications:

3- Une problèmatique en physique trouve sa solution uniquement en la modélisant.

$\begin{array}{ll}\text { a- } & \text { Oui } \\ \text { b- Non } \\ \text { c- Autres réponses ou justifications: }\end{array}$

4- Par ordre de préférence, choisissez 04 définitions de la notion de modèle avec lesquelles vous vous sentez le plus en accord (1 pour la meilleure proposition puis 2,3 et 4).

a- Une représentation simplifiée de la réalité d'un phénomène.

b- Une représentation idéale de la réalité d'un phénomène.

c- Une formulation mathématique.

d- Un schéma simplificateur

e- Une hypothèse.

f- Un moyen de représentation utilisée pour décrire et expliquer divers phénomènes.

g- Une matérialisation des énoncés de la science dans un objet concret.

h- Un modèle est un système, conçu comme une représentation abstraite et utile d'un autre système.

i- Autre réponse ou justification:

5- Par ordre de préférence, choisissez 04 rôles des modèles avec lesquels vous vous sentez le plus en accord (1 pour la meilleure proposition puis 2,3 et 4):

a- Prévoir avec une plus grande probabilité et une meilleure certitude, différents comportements ou différentes situations.

b- Donner la description des phénomènes.

c- Donner l'explication des phénomènes.

d- Avoir une fonction d'investigation et de mesure.

e- Aider à la perception et à la représentation des phénomènes.

f- Mettre en relation des concepts explicitement définis.

g- Aider à concevoir, à analyser ou à imaginer des concepts.

h- Aider à la conception d'hypothèses et d'expériences.

i- Autre réponse ou justification: 
6- A votre avis ; les étudiants sont-ils aptes à concevoir la notion de "modèle" ?
a- Oui.
b- Non.

7- Y'a-t-il un intérêt de définir ou de caractériser aux étudiants la notion de modèle et modélisation?
a- Oui.
b- Non.
c- Peu importe.

8- Pensez-vous que les méthodes d'enseignement actuelles permettent à l'étudiant de se construire la notion du "modèle" ?
a- Oui.
b- Non.
c- Commentaire ou justification

9- Dans vos enseignements avez-vous utilisé des modèles différents ou contradictoires pour un même phénomène?
a- Oui
b- Non
c- $\quad$ Autre réponse ou justification

10- Quelle stratégie adoptez-vous pour clarifier un modèle scientifique quelconque (vous pouvez choisir plusieurs stratégies avec lesquelles vous êtes le plus d'accord).

a- Exposer le modèle en détail aux étudiants qui doivent se l'approprier.

b- Procéder par présentation historique.

c- Élaborer le modèle à partir des réponses des apprenants avec leurs mots et leurs phrases.

d- Recourir à l'épistémologie du modèle.

e- Analyser et interpréter le modèle à partir d'un problème situé dans son contexte.

f- Guider les apprenants à élaborer eux-mêmes le modèle. 


\begin{tabular}{|c|c|c|c|c|c|c|c|c|c|c|c|c|c|c|c|c|c|}
\hline 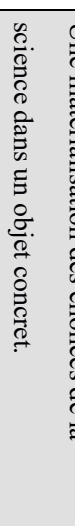 & 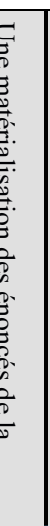 & 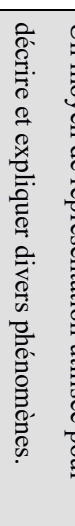 & 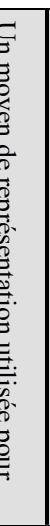 & & 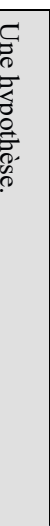 & & 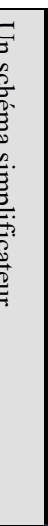 & 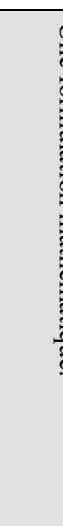 & 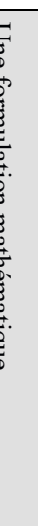 & 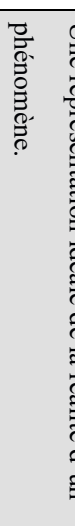 & 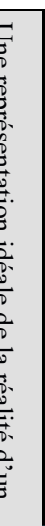 & 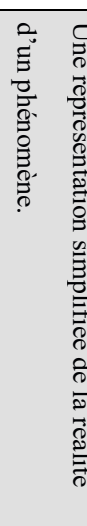 & & & & & 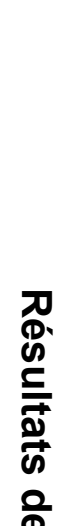 \\
\hline के & $N$ & $\begin{array}{l}\vec{t} \\
\stackrel{\Delta}{\circ}\end{array}$ & $\overrightarrow{u_{1}}$ & $\stackrel{8}{\circ}$ & 0 & $\stackrel{\circ}{\circ}$ & 0 & $\stackrel{\circ}{\circ}$ & $\omega$ & के & $N$ & $\underset{\frac{\omega}{d}}{\frac{\omega}{a^{\circ}}}$ & $\bar{N}$ & $\Theta$ & \multirow{4}{*}{\multicolumn{2}{|c|}{ 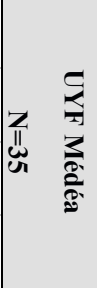 }} & $\frac{1}{F}$ \\
\hline$\stackrel{\omega}{\circ}$ & - & 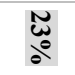 & $\infty$ & $\frac{\hat{\theta}}{0}$ & $N$ & aे & $N$ & ă & 0 & $\frac{a}{0}$ & $N$ & $\begin{array}{l}\tilde{\omega} \\
\text { de }\end{array}$ & $\infty$ & ని & & & $\frac{\mathbb{D}}{3}$ \\
\hline $\overrightarrow{0}$ & \pm & $\frac{\vec{t}}{\partial^{\circ}}$ & $u$ & : & 0 & $\begin{array}{l}\tilde{u} \\
\stackrel{N}{0} \\
\partial^{\circ}\end{array}$ & $\infty$ & $\begin{array}{l}\tilde{u} \\
\stackrel{\tilde{N}}{\circ}\end{array}$ & $\infty$ & $\stackrel{w}{\circ}$ & - & $\frac{\vec{E}}{a^{\circ}}$ & $u$ & ఓు & & & $\begin{array}{l}N \\
\ddot{r}\end{array}$ \\
\hline$\frac{E}{\partial^{\circ}}$ & ur & $\stackrel{\circ}{\circ}$ & $\omega$ & $\stackrel{\circ}{\circ}$ & $\omega$ & $\frac{5}{20}$ & + & $\stackrel{\circ}{\circ}$ & $\omega$ & $\stackrel{0}{\circ}$ & $\omega$ & $\overrightarrow{0}$ & + & 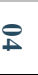 & & & 응 \\
\hline$\stackrel{0}{\circ}$ & 0 & : & 0 & $\stackrel{8}{\circ}$ & 0 & 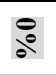 & 0 & $\frac{\bar{J}}{\sigma^{\circ}}$ & - & $\frac{f}{a^{\circ}}$ & + & $\frac{\vec{t}}{\theta^{\circ}}$ & + & $\Theta$ & \multirow{4}{*}{\multicolumn{2}{|c|}{ 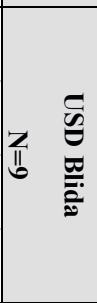 }} & $\stackrel{\text { D }}{\text { D }}$ \\
\hline$\stackrel{8}{\circ}$ & 0 & $\frac{\mathfrak{N}}{a^{\circ}}$ & $N$ & $\stackrel{\circ}{\circ}$ & 0 & $\stackrel{0}{\circ}$ & 0 & $\begin{array}{l}\stackrel{\vec{W}}{\mathbf{J}} \\
\partial^{\circ}\end{array}$ & $\omega$ & $\frac{\mathbb{N}}{\mathbb{N}^{\circ}}$ & $N$ & $\frac{0^{\circ}}{\mathfrak{N}}$ & $N$ & ㄱ. & & & 흘 \\
\hline$\frac{\vec{b}}{20}$ & - & $\begin{array}{l}\mathscr{\omega}_{\vec{J}} \\
\partial^{\circ}\end{array}$ & $\omega$ & $\stackrel{8}{\circ}$ & 0 & $\frac{\bar{a}}{0^{\circ}}$ & - & 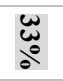 & $\omega$ & $\stackrel{8}{\circ}$ & 0 & $\frac{\overrightarrow{0}}{\circ}$ & - & ¿ & & & 을 \\
\hline$\frac{5}{0}$ & - & 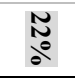 & $N$ & $\frac{5}{2}$ & - & $\frac{0^{\circ}}{\mathrm{N}}$ & $N$ & $\frac{0^{\circ}}{N}$ & N & $\stackrel{8}{\circ}$ & 0 & $\stackrel{8}{\circ}$ & 0 & 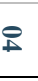 & & & 3 \\
\hline पू & - & $\vec{t}$ & $\omega$ & $\therefore$ & 0 & $\stackrel{\circ}{\circ}$ & $N$ & $\stackrel{\circ}{\circ}$ & $N$ & $\stackrel{\infty}{\circ}$ & 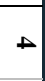 & 峁 & $\infty$ & $\Theta$ & \multirow{4}{*}{\multicolumn{2}{|c|}{ 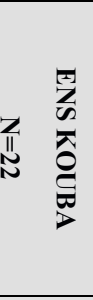 }} & $\frac{\overline{0}}{\mathrm{D}}$ \\
\hline$\stackrel{0}{\circ}$ & $N$ & $\frac{\vec{E}}{d^{\circ}}$ & $\omega$ & $\frac{11}{0^{\circ}}$ & - & $\frac{\vec{E}}{\partial^{\circ}}$ & $\omega$ & $\frac{\vec{E}}{\partial^{\circ}}$ & w & पI & - & $\frac{\tilde{\omega}}{\sigma^{\circ}}$ & ur & న & & & $\overparen{7}$ \\
\hline$\frac{\vec{f}}{\sigma^{\circ}}$ & $\omega$ & 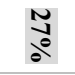 & $a$ & $\stackrel{8}{\circ}$ & 0 & $\stackrel{0}{\circ}$ & $N$ & $\stackrel{\circ}{\circ}$ & $N$ & $\stackrel{\circ}{\circ}$ & $N$ & $\stackrel{\circ}{\circ}$ & 0 & జు & & & 을 \\
\hline$\stackrel{0}{\circ}$ & $N$ & $\stackrel{\circ}{\circ}$ & 0 & $\frac{\text { un }}{0^{\circ}}$ & - & $\stackrel{0}{0}$ & $N$ & $\stackrel{\text { I }}{\text { Na }}$ & $a$ & $\stackrel{\circ}{\circ}$ & 0 & $\begin{array}{l}\vec{\infty} \\
\dot{0}\end{array}$ & + & $\Phi$ & & & $\frac{\text { D }}{2}$ \\
\hline$\stackrel{N}{0}$ & - & $\begin{array}{l}\underset{\omega}{w} \\
\stackrel{\omega}{\circ}\end{array}$ & $\vec{A}$ & $\frac{8}{8}$ & 0 & Uू & $N$ & aे & $\omega$ & 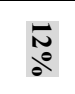 & ur & $\frac{\omega}{\partial}$ & $\bar{u}$ & $\Theta$ & \multirow{4}{*}{\multicolumn{2}{|c|}{ 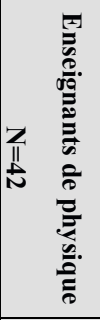 }} & $\begin{array}{l}\text { (1) } \\
\text { \& } \\
0\end{array}$ \\
\hline$\frac{\text { ug }}{0^{\circ}}$ & $N$ & ఫे & $\checkmark$ & $\stackrel{N}{\circ}$ & - & ¿ै & $\omega$ & $\frac{n}{D^{0}}$ & $\Xi$ & $\frac{\overrightarrow{8}}{\circ}$ & + & $\frac{N}{8}$ & 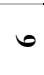 & న & & & 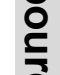 \\
\hline हे & + & वे & $\checkmark$ & $\stackrel{8}{\circ}$ & 0 & $\frac{\vec{E}}{\partial 0}$ & $a$ & $\frac{a^{\circ}}{n}$ & $\theta$ & ¿ै & $\omega$ & $\frac{\vec{t}}{0^{\circ}}$ & $a$ & $\ddot{\omega}$ & & & $\stackrel{\overbrace{}}{\mathbb{D}}$ \\
\hline ह & + & वै & $\omega$ & ¿ै & $\omega$ & $\vec{\partial}$ & $v$ & $\frac{\vec{t}}{\partial^{\circ}}$ & $a$ & ¿ै & $\omega$ & $\stackrel{d}{a}$ & $\omega$ & $\Phi$ & & & ڤి \\
\hline$\stackrel{\infty}{0}$ & $N$ & बे & \pm & $\stackrel{8}{\circ}$ & 0 & $\stackrel{8}{\circ}$ & 0 & $\begin{array}{l}\bar{\omega} \\
\stackrel{\sigma}{\circ}\end{array}$ & $\omega$ & $\frac{N}{0}$ & u & $\begin{array}{l}\mathscr{U}_{\infty} \\
\stackrel{0}{0}\end{array}$ & 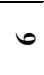 & $\Theta$ & \multirow{4}{*}{\multicolumn{2}{|c|}{ 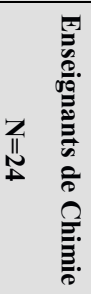 }} & \\
\hline$\frac{\overrightarrow{0}}{\partial 0}$ & - & $\begin{array}{l}\tilde{U} \\
\text { di }\end{array}$ & $a$ & $\stackrel{\infty}{0}$ & $N$ & $\stackrel{\infty}{0^{0}}$ & $N$ & $\frac{N}{a^{\circ}}$ & ur & $\frac{\overrightarrow{0}}{20}$ & - & $\frac{\tilde{u}}{0}$ & $a$ & ని & & & \\
\hline ¿े & + & $\frac{\mathfrak{d}}{\mathfrak{U}^{\circ}}$ & $v$ & $\stackrel{8}{\circ}$ & 0 & $\frac{N}{2}$ & u & $\begin{array}{l}\vec{\omega} \\
\partial^{\circ}\end{array}$ & $\omega$ & $\stackrel{\circ}{\circ}$ & 0 & $\stackrel{0}{\circ}$ & 0 & ఓు & & & \\
\hline$\vec{a}$ & + & $\stackrel{\infty}{\rho^{\circ}}$ & $N$ & $\stackrel{\infty}{\theta^{\circ}}$ & $N$ & $\frac{\vec{b}}{\partial^{\circ}}$ & - & $\frac{\pi}{2}$ & u & $\stackrel{8}{\circ}$ & 0 & $\frac{N}{\sigma^{\circ}}$ & u & $\stackrel{8}{1}$ & & & \\
\hline
\end{tabular}




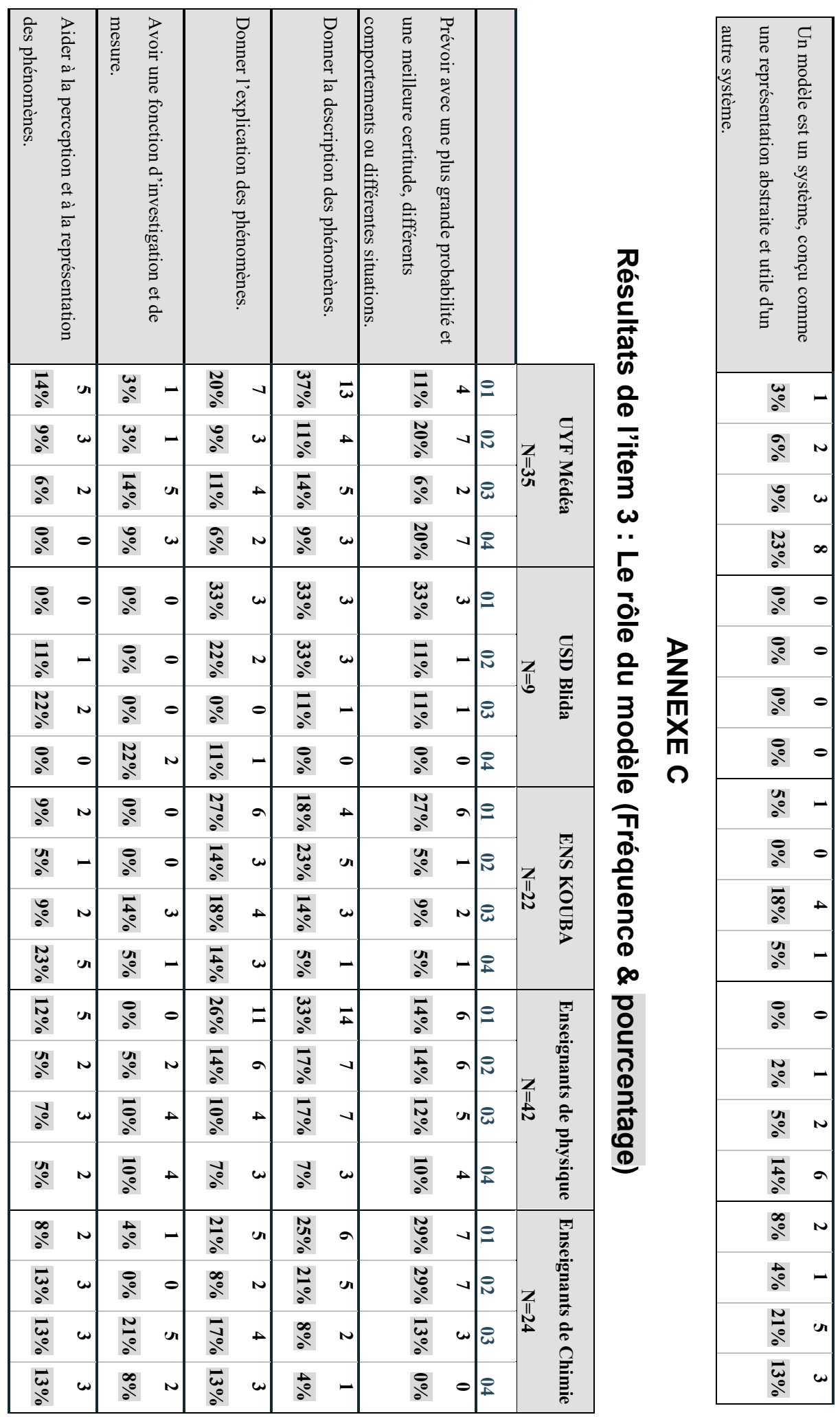




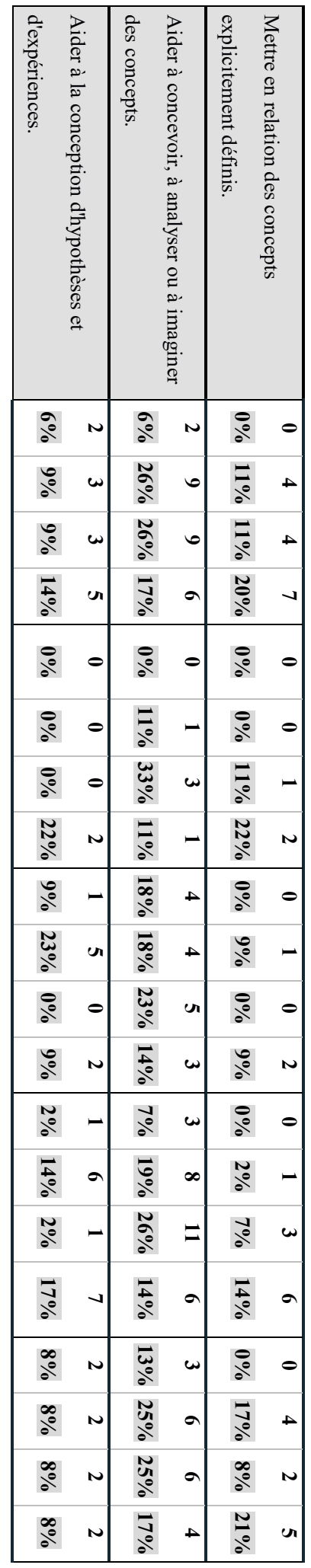

